\title{
Analysis of the Ultrastructure of the Canine Zona Pellucida
}

\author{
M.O. Lunn,* and S.J. Wright* \\ * Department of Biology, University of Dayton, Dayton, OH 45469-2320.
}

Mature mammalian oocytes are surrounded by an extracellular matrix called the zona pellucida that sperm penetrate during fertilization $[1,2]$. The zona pellucida is composed of three different glycoproteins that form a fibrous three-dimensional network of filaments $[1,2]$. The zona pellucida first appears during folliculogenesis and surrounds the oocyte and early embryo until the blastocyst embryo "hatches" from the zona pellucida $[1,2]$. The zona pellucida plays a critical role in species-specific sperm binding to the oocyte, and prevents polyspermy. It also mechanically protects the oocyte and embryo, prevents premature adherence to the female reproductive system, and protects the embryo from rejection by the immune system [cf. 3]. Although the structure and function of the zona pellucida of several mammals such as mice, cows and the human have been well studied, little is known about the morphology of the zona pellucida of companion animals. The objective of the study was to elucidate the ultrastructure of the canine (Canis familiaris) zona pellucida by scanning electron microscopy (SEM). Ovaries were collected from 29 different dog breeds aged 3 months to 9 years. Ovaries were minced to release cumulus-oocyte complexes which were physically stripped of cumulus cells using a small bore pipet. We investigated the surface morphology of 305 zona intact oocytes for the study. The oocytes were fixed for $1 \mathrm{hr}$ in $2.5 \%$ glutaraldehyde in $0.1 \mathrm{M}$ sodium cacodylate buffer, $\mathrm{pH}$ 7.4 during affixation to $1 \mathrm{mg} / \mathrm{ml}$ poly-L-lysine coated glass slides. The oocytes were critical point dried in a Tousimis Autosamdri 814B critical point dryer, sputter coated with gold in a Denton Vacuum Desk II sputter coater, and viewed with a JEOL JSM-5800LV scanning electron microscope at $15 \mathrm{kV}$ accelerating voltage with a $11 \mathrm{~mm}$ working distance at low $(1,000 \mathrm{X})$ and high magnification $(4,000 \mathrm{X})$. When measured from SEM micrographs, the oocytes ranged in diameter from approximately 50 to $110 \mu \mathrm{m}$. In many of the oocytes, the zona pellucida appeared as a fibrous network with various hollows and pores; however, it was unexpectedly heterogeneous. Because of this, oocytes were placed into one of four groups based on the appearance of their zona pellucida at low and high magnification (Fig. 1). In oocytes with a Type I zona pellucida, the zona pellucida was smooth at both low and high magnification and had no pores or a few small $(0.5 \mu \mathrm{m})$ pores at high magnification. Oocytes with the Type II zona pellucida had a smooth appearance at low magnification, and at high magnification, the zona pellucida was multi-layered. The outer layer appeared spongy, uniform, and fenestrated with numerous pores that were oval or spherical in shape. Oocytes with a Type III zona pellucida were characterized by a rough, "shaggy" appearance at low magnification. At high magnification, the outer surface was uneven with raised areas and usually was fenestrated with pores or hollows of varying sizes. The Type IV zona pellucida also appeared "shaggy" at low magnification; however, at high magnification the Type IV zona pellucida had numerous thin fibrils throughout its surface. Most of the oocytes $(n=201)$ had a rough-appearing zona pellucida of Type III $(n=113)$ or Type IV $(n=88)$. Fewer oocytes had a smooth Type II zona pellucida $(n=93)$ and very few had a Type I zona pellucida $(n=11)$. Closer examination of the pores from a typical Type II oocyte revealed that the pores were conical narrowing as they progress through the zona pellucida (Fig. 2). Some pores bifurcated into smaller pores at deeper depths that did not appear to completely penetrate the zona pellucida, suggesting that the zona 
pellucida may prevent viruses, such as the canine distemper virus from penetrating the zona pellucida and infecting the oocyte/embryo. In cases where some of the cumulus cells remained attached to the zona pellucida, the cumulus cells had a single narrowing protrusion that penetrated into the zona pellucida similar to what has been reported in human oocytes [4]. We conclude that canine oocytes from the same dog as well as different dogs have a highly heterogeneous ultrastructure. This heterogeneity does not appear to be due to individual dogs, dog breed, age or size of the canine oocyte donor, and oocyte maturity (as reflected in oocyte size) since each of these parameters had multiple oocytes of almost every type [5].

\section{References}

[1] B.S. Dunbar et al., A Comparative Overview of Mammalian Fertilization, Plenum, New York, 1991.

[2] B.S. Dunbar et al., Reprod. Fertil. Dev. 6 (1994) 331.

[3] R.C. Ribas et al., Biol. Reprod. 74 (2006) 307.

[4] Magerkurth et al., Human Reprod. 14 (1999) 1057.

[5] Funded by the Canine Health Foundation of the American Kennel Club.

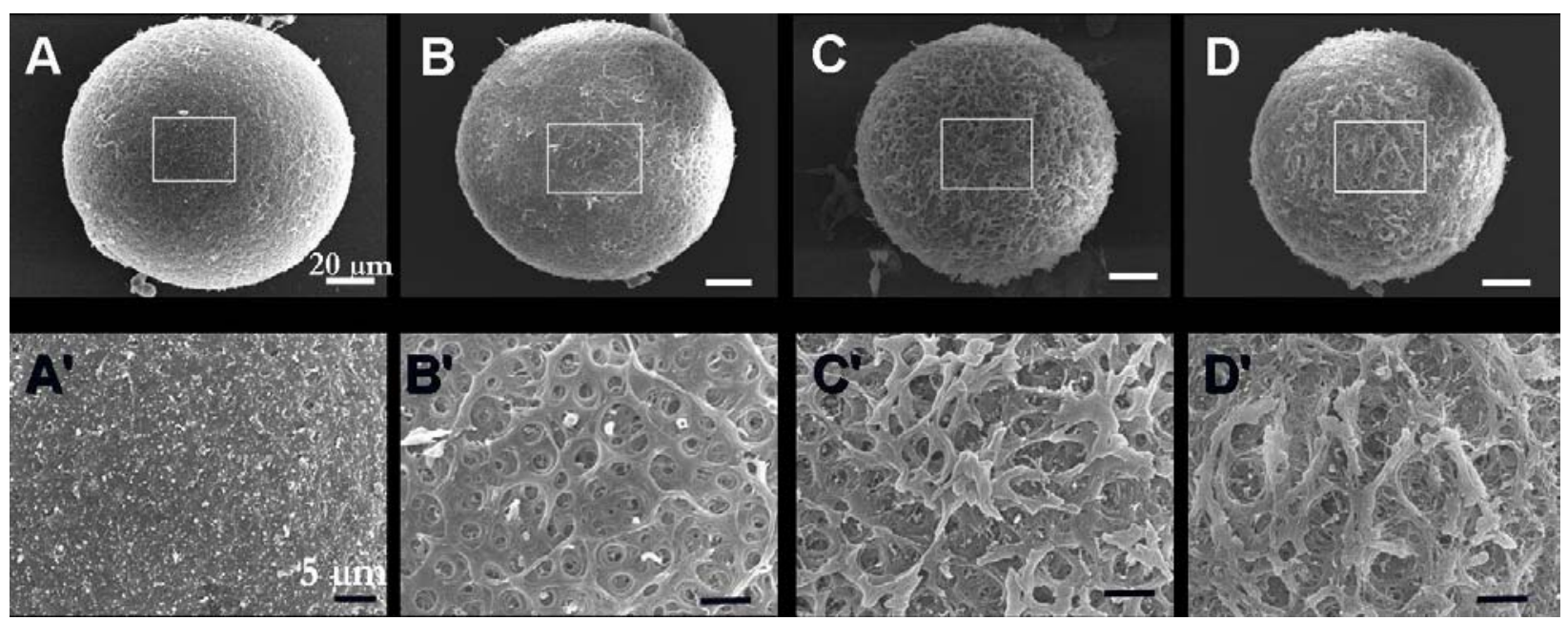

FIG. 1. Scanning electron micrographs of canine oocytes showing the heterogeneity of zona pellucida structure. Type I, A, A'; Type II, B, B'; Type III, C, C'; Type IV, D, D'. A, C, 950x; B, D, 1,000x; A'-D', 4,000x. Scale bars A-D, $20 \mu \mathrm{m}, \mathrm{A}^{\prime}-\mathrm{D}^{\prime}, 5 \mu \mathrm{m}$.

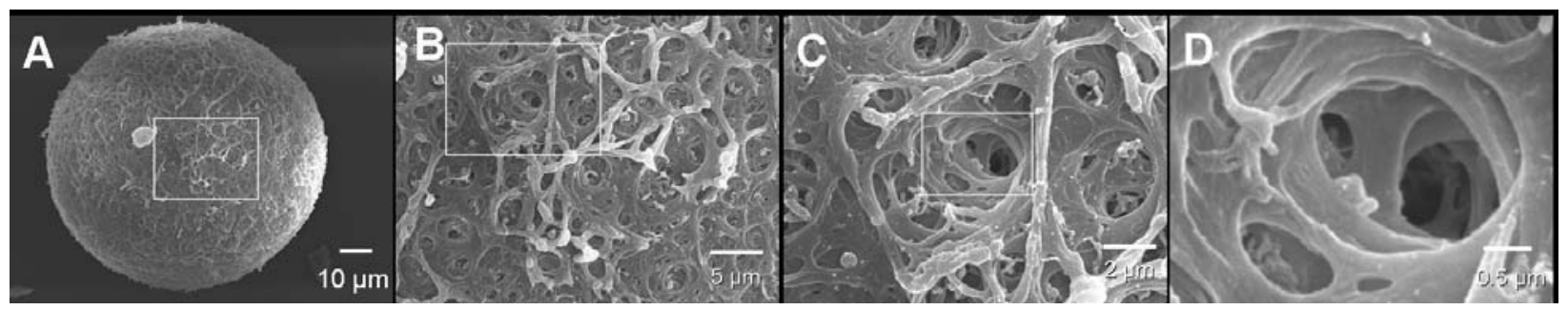

FIG. 2. Scanning electron micrographs of a canine oocyte at increasingly higher magnifications show that zona pellucida pores can be conical and bifurcated. A, 1,100x; B, 4,000x; C, 10,000x; D, 35,000x. Scale bars: A, $10 \mu \mathrm{m}$; B, $5 \mu \mathrm{m}$; C, $2 \mu \mathrm{m}$; D, $0.5 \mu \mathrm{m}$. 